\title{
A invisibilidade das mulheres na Física: um recorte nos últimos 12 anos na produção de eventos e revistas de alto impacto
}

\author{
Camila Andrade Pandini ${ }^{\star}$, Roberta Chiesa Bartelmebs ${ }^{\star \star}$, Maria Milena Figueira Tegon ${ }^{\star \star \star}$
}

\section{Resumo}

A participação das mulheres nas carreiras científicas, tem sido um tema muito recorrente nos estudos atuais. Pesquisas têm mostrado que em determinadas áreas, a participação feminina tem aumentado e até ultrapassado homens, mas isso não se aplica ao caso da Física. Um dos desafios para a maior representatividade de mulheres na carreira de Física, é causado por ser uma área considerada essencialmente masculina por muitos, o que resulta na desmotivação de meninas em seguir neste campo. Entendemos que uma das maneiras de mudar esta visão de mundo sobre as mulheres na Física, é a maior representatividade delas em revistas ou eventos científicos da área. O presente artigo faz uma revisão de modo a verificar se houve, nos últimos 12 anos, de 2008 a 2020, algum aumento desta representação. Para isso, selecionou-se cinco revistas e cinco eventos nacionais e internacionais de alto impacto. Os dados recolhidos revelam uma discrepância bastante alta na quantidade de publicação entre homens e mulheres. Portanto esse trabalho demonstra, que ainda há muito a ser feito na área da Física para que haja uma equidade na produção científica e na participação feminina nas publicações da área.

Palavras-chave: Mulheres; Física; Publicações.

* Graduanda do curso de Licenciatura em Ciências Exatas na habilitação de Física pela Universidade Federal do Paraná - Setor Palotina. E-mail:camila.pandini@ufpr.br. ORCID: https://orcid.org/0000-0002-3227-0262

* Doutora em Educação em Ciências e Matemática pela Pontifícia Universidade Católica do Rio Grande do Sul. Docente do Programa de Pós-Graduação em Ensino de Ciências, Ensino de Matemática e Tecnologias Educativas. E-mail: roberta.bartelmebs@ufpr.br. ORCID: https://orcid.org/0000-0002-1057-6623

*** Mestranda do Programa de Pós-Graduação em Ensino de Ciências, Ensino de Matemática e Tecnologias Educativas na Universidade Federal do Paraná-Setor Palotina. Licenciada em Ciências Exatas com habilitação em Física. Email: milenategon@ufpr.br. ORCID: https://orcid.org/0000-0003-2463-8941 


\section{Introdução}

Por muito tempo a ciência foi considerada um conhecimento que só os homens poderiam compreender, sendo as mulheres tidas como incapazes. Ainda hoje vemos esses traços deixados pelo passado, como as mulheres sendo minoria na ciência, mesmo que em muitas áreas esteja ocorrendo um crescimento, há uma área em que este aumento está acontecendo de forma lenta em comparação às outras, sendo essa a área da Física.

De acordo com Bolzani (2017), em 2013, o governo dos Estados Unidos disponibilizou dados sobre as ocupações das mulheres na área de Science, Technology, Engineering and Mathematics (STEAM). De acordo com estes dados, as mulheres constituíam $46 \%$ da força de trabalho no país, apesar disso, apenas $27 \%$ delas ocupavam os postos em ciência e engenharia e $12 \%$ no segmento exclusivo de engenharia. No Brasil, em 2012, os dados revelam que nos cursos de graduação, considerando-se todas as carreiras, $57,1 \%$ das mulheres foram formadas em áreas onde a predominância feminina é marcante - como pedagogia, letras, ciências humanas (BOLZANI, 2017). Estes dados nos revelam que as mulheres conseguiram conquistar um espaço na sociedade, porém, este espaço ainda está voltado para as áreas tradicionalmente tida como femininas, voltadas ao cuidado e ao ensino.

Se fecharmos mais o leque da área das exatas a Física está entre um dos campos em que as mulheres menos aparecem historicamente, e quando aparecem estão sub-representadas, sendo esta uma das possíveis respostas do porque o crescimento das mulheres nessa área está caminhando tão lentamente (CORDEIRO, 2017). Assim, uma das maneiras de mudar este cenário é aumentar o número da participação das mulheres na Física, buscando representá-las, visto que várias jovens com grande potencial intelectual são desencorajadas ou sentem que não estão aptas à estudar Física.

Desse modo, este trabalho, fruto de uma pesquisa de trabalho de conclusão de curso realizado na Universidade Federal do Paraná, tem como objetivo constatar se nestes últimos 12 anos houve um crescimento na participação das mulheres na Física. Para tanto, realizamos um levantamento bibliográfico em cinco revistas de alto impacto e em cinco cadernos de resumos de eventos de impacto internacional na área da Física. 


\section{As mulheres cientistas: reflexões históricas e sociais}

Ao olhar para o passado é possível notar que nas áreas que envolvem as Ciências, principalmente a de Exatas, há uma predominância masculina. Isso pode ser consequência das convenções sociais que até início do século XX consideravam que era inadequado para as mulheres ocupar uma posição de destaque profissional.

Tal situação chegou a ser justificada pela determinação biológica (ANDRADE, 2011). Muitas vezes são preconceitos que passam de geração em geração e se encontram enraizados em nossa cultura, condicionando o comportamento feminino e sua posição na sociedade. Durante muitos anos as mulheres foram impedidas de participar dos ambientes acadêmicos, e com muita dificuldade conseguiram ocupar espaço na Ciência, porém de forma desigual e sem o devido reconhecimento (ALMEIDA, 2017).

É facilmente visualizado que ainda vivemos em uma civilização com viés masculino. Um exemplo disso é a lista dos 100 nomes, The One Hundred (HART, 1996), uma lista das pessoas consideradas as mais significativas em termos de influências na história da humanidade. O resultado é uma lista com 98 nomes de homens e apenas duas mulheres, sendo estas duas rainhas: Isabel, a Católica (n. 65), e Elizabeth I (n. 94) (CHASSOT, 2004).

Os principais obstáculos que as mulheres enfrentam na Ciência estão relacionados às dificuldades em conciliar a carreira profissional com a vida pessoal. Outro ponto é a discriminação no ambiente de trabalho, ao isolamento profissional e a pequena representatividade de mulheres em todos os níveis de decisão. A esse respeito, Cartaxo (2012, p.17) complementa:

[...]. A questão é que os homens não são “cobrados ou responsabilizados” pela vida privada do lar, socialmente, e para muitos essa ainda é uma atividade de responsabilidade única e exclusivamente da mulher. $\mathrm{O}$ fato é que a sociedade mudou, as mulheres trabalham tanto quanto os homens, mas aos olhos da sociedade, a criação dos filhos e a organização da casa continuam sendo uma responsabilidade da mulher. De fato, a literatura especializada e as conversas com as mulheres físicas entrevistadas apontam que se as mulheres querem fazer ciência, precisam se comportar tal como um homem, ou escolher o que é mais importante para elas: a carreira ou a vida particular e familiar.

Além da pressão pela responsabilização da vida do lar, as mulheres, muitas vezes, estão sub-representadas na Ciência, especialmente na Física, refletindo a 
visão estereotipada de um cientista, resultando na falta de um modelo em que as meninas e mulheres possam se espelhar. Para quebrar tais estereótipos é necessário resgatar as figuras femininas caídas no esquecimento e aquelas ocultas da história da Ciência, para que as novas gerações tenham a quem se refletir (MELO; RODRIGUES, 2013).

Uma das únicas mulheres cientistas que continua sendo citada nas aulas é Madame Marie Curie. Isso evidencia de uma maneira subliminar para as meninas, que apenas poucas mulheres são ditas inteligentes o suficiente para conseguir se dar bem nas Ciências. Assim, muitas vezes quando elas se mostram capazes, são ditas esforçadas, já quando os meninos têm aptidão para ciências, são inteligentes. Mesmo que se saiba que essa é uma falsa premissa, ainda hoje a situação é reforçada (CHASSOT, 2004; SCHIEBINGER, 2008; SILVA; SANTOS; HEERDT, 2017).

\section{As imagens das mulheres nos materiais didáticos de ciências}

Com relação ao contexto educacional, Martins e Hoffmann (2007) analisaram as imagens de livros didáticos de Ciências, do Ensino Fundamental. Os autores encontraram em seus resultados que $43 \%$ dos livros analisados, aparecem mulheres realizando alguma tarefa doméstica. 53\% dos livros apresentam imagens de mulheres que são voltados à culinária e em $82 \%$ dos livros, as mulheres aparecem cuidando do marido e das crianças.

Nas imagens em que as mulheres aparecem em profissionais os resultados foram os seguintes: $45 \%$ são professoras, $25 \%$ enfermeiras e odontólogas e $14 \%$ em outros trabalhos. Nas imagens que aparecem os homens, $16 \%$ aparece cuidando de crianças, $5 \%$ ajudando em tarefas domésticas e $9 \%$ no preparo da alimentação.

Esses dados mostram que a ideia de que a mulher deve trabalhar com algo voltado ao cuidar é reforçado por imagens que as crianças vêem em sua educação. Em sua pesquisa, Ginity (2015) ao analisar as obras didáticas contempladas pelo Plano Nacional do Livro Didático (PNLD) de 2015, apresenta que as representações imagéticas dos livros ainda contribuem "[...] na composição de identidades que dão prosseguimento a determinadas visões de mundo, estimuladas pelos interesses de grupos culturais, socioeconômicos e políticos" (GINITY, 2015, p. 915). 


\section{A exclusão das mulheres do trabalho científico: metáforas para compreender um fenômeno antigo}

A exclusão das mulheres na Ciência foi mapeada de forma "horizontal ou territorial, que se refere à exclusão das mulheres do topo da carreira científica e a vertical ou hierárquica, que trata de áreas do conhecimento caracterizadas em femininas ou masculinas na ciência" (LIMA, 2008). A exclusão vertical também é conhecida pelo fenômeno "Teto de Vidro", metáfora para a invisibilidade das barreiras que impedem ou dificultam o progresso das mulheres na carreira (SILVA; RIBEIRO, 2014).

Outra metáfora importante é a do Efeito Matilda (ROSSITER, 1993). Segundo Benedito (2019), o efeito Matilda faz referência ao fato de que o trabalho de pesquisadoras mulheres não tem o mesmo reconhecimento do que o de seus colegas homens. Assim: "De tão recorrente, o fenômeno ganhou nome: 'efeito Matilda', em uma referência à Matilda Joslyn Gage, ativista pelo sufrágio universal, abolicionista e pensadora americana" (BENETIDO, 2019, p. 8).

Vasconcellos e Brisola (2009), iniciaram um levantamento sobre a minoria de estudantes e docêntes do sexo feminino na Universidade Estadual de Campinas (Unicamp) em disciplinas concideradas "masculinas", que incluem as ciências exatas e tecnológicas ou engenharias, identificando que não existe diferenças por sexo na capacidade de aprendizado e na dedicação dos alunos, segundo as auutoras:

Há, no entanto, uma evidência clara nos dados, apontando para o fato de que homens e mulheres são igualmente capazes de seguir carreiras das ciências mais diversas. O maior fluxo de homens para ciências exatas e tecnológicas se deve a questões de fundo cultural e social e não às possíveis capacidades inatas diferenciadas por sexo. Além do condicionamento social, desempenham papel importante na escolha das carreiras as práticas ainda discriminatórias que afastam mulheres de profissões consideradas masculinas. O próprio ambiente de trabalho, nesses casos em que a presença feminina é consideravelmente reduzida, de certa forma, afasta a mulher e favorece atitudes sexistas (VASCONCELLOS; BRISOLLA, 2009, p. 249).

De acordo com Menezes (2017):

A Sociedade Brasileira de Física (SBF) possui $27 \%$ de mulheres filiadas e a Sociedade Astronômica Brasileira (SAB) conta com $29 \%$ de mulheres. No entanto, o percentual de mulheres bolsistas é de apenas $11 \%$, um terço do valor que corresponde à representatividade das mulheres nas áreas de Física e Astronomia (MENEZES, 2017, p. 342). 
Essa situação persiste há mais de 15 anos. Embora não tenhamos ainda dados estritamente objetivos para tal situação, é possível inferir que ele está diretamente ligado ao fato de as pesquisadoras serem em menor número entre os Bolsistas Produtividade do Conselho de Pesquisa e Pós-Graduação (CNPq).

E por fim, um estudo elaborado por Nunes et al. (2020), demonstra que ainda é baixo o número de mulheres cientistas brasileiras que são reconhecidas pelos alunos e alunas da Educação Básica. Segundo os autores: “[...] tal fato pode acarretar no desinteresse das mulheres em ingressarem em carreiras científicas futuramente" (p. 86220).

Em uma palestra, Barbosa (2010), aponta que a Física necessita das mulheres, pois os problemas apresentados na Física do século XXI, são de um alto grau de complexidade e necessitam da colaboração de muitos profissionais de qualidade. Dessa forma, a Física precisa contar com $100 \%$ da população, para ter disponível as melhores mentes, o que significa dizer que tornar a Física da complexidade uma ciência exclusivamente masculina pode condenar a área a não ter a evolução que ela mereceria.

Entendemos que uma das formas de motivar meninas a seguirem carreira na Física seja a representatividade feminina na área, para tanto, desenvolveu-se esta pesquisa, com o intuito de revelar um panorama geral da participação de pesquisadoras em publicações de artigos em anais de eventos e revistas de alto impacto.

\section{Metodologia}

Este trabalho é uma pesquisa qualitativa de cunho bibliográfico. De acordo com Gibbs (2009), a pesquisa qualitativa busca investigar a maneira como as pessoas constroem o mundo a sua volta, além disso, os documentos e as interações são considerados como forma de estabelecer, de forma conjunta ou conflituosa, processos e artefatos sociais que podem ser reconstruídos e analisados com diversos métodos qualitativos que permitem ao pesquisador criar modelos, teorias, como formas de descrever e explicar as questões sociais.

Para Boccato (2006), a pesquisa bibliográfica visa a resolução de um problema por meio de referenciais teóricos publicados, analisando e discutindo as várias contribuições científicas. Buscando assim trazer subsídios para o conhecimento sobre o tema investigado. 
Esta pesquisa foi realizada analisando as publicações de cinco revistas de impacto internacional da área de Física e cinco dos eventos da mesma área, também com impacto internacional. No quadro a seguir apresentamos as revistas e eventos selecionados para esta pesquisa:

Quadro 1: Revistas e eventos utilizados na investigação

\begin{tabular}{|l|l|l|}
\hline \multicolumn{1}{|c|}{ Título } & \multicolumn{1}{|c|}{ Site acesso } & Abrangência \\
\hline $\begin{array}{l}\text { Encontro Nacional de Física } \\
\text { da Matéria Condensada }\end{array}$ & http://www.sbfisica.org.br/ enfmc/xxxii// & Nacional \\
\hline $\begin{array}{l}\text { International Symposium on } \\
\text { Lepton Photon Interactions at } \\
\text { High Energies }\end{array}$ & $\begin{array}{l}\text { https://www-conf.slac.stanford.edu/lp13/partici- } \\
\text { pant_list.asp }\end{array}$ & Internacional \\
\hline $\begin{array}{l}\text { International Symposium on } \\
\text { Very High Energy Cosmic Ray } \\
\text { Interactions }\end{array}$ & $\begin{array}{l}\text { https://g3indico.tifr.res.in/event/598/registrations/ } \\
\text { participants }\end{array}$ & Internacional \\
\hline $\begin{array}{l}\text { International Conferences on } \\
\text { Precision Physics of Simple } \\
\text { Atomic Systems (PSAS) }\end{array}$ & http://psas2020.csp.escience.cn/dct/page/70051 & Internacional \\
\hline Solvay Conference on Physics & $\begin{array}{l}\text { http://www.solvayinstitutes.be/html/solvayconf_- } \\
\text { physics.html }\end{array}$ & Internacional \\
\hline \multicolumn{1}{|c|}{ Título } & \multicolumn{1}{|c|}{ Revistas } & Site acesso \\
\hline Abrangência \\
\hline Brazilian Journal of Physic & $\begin{array}{l}\text { https://link.springer.com/journal/13538/volumes- } \\
\text {-and-issues }\end{array}$ & Nacional \\
\hline Reviews of modern Physic & https://journals.aps.org/rmp/issues & Internacional \\
\hline Physical Review X & https://journals.aps.org/prx/issues & Internacional \\
\hline Annalen der Physik & https://onlinelibrary.wiley.com/loi/15213889 & Internacional \\
\hline Nature Physics & https://www.nature.com/nphys/volumes & Internacional \\
\hline
\end{tabular}

Fonte: Dados das autoras (2021).

\section{Resultados e discussões}

Dividimos a apresentação e discussão dos resultados em dois momentos. No primeiro, apresentaremos os dados relativos à participação das mulheres nos eventos da área da Física. A seguir, apresentaremos os dados das publicações nas revistas selecionadas. 


\section{Participação das mulheres em eventos}

Para compreendermos se houve um crescimento na participação das mulheres em eventos relacionados à Física analisamos as suas produções nos anais dos eventos do Quadro 2 a seguir.

Quadro 2: Eventos pesquisados

\begin{tabular}{|l|c|}
\hline \multicolumn{1}{|c|}{ Eventos } & Anos dos anais pesquisados \\
\hline Encontro Nacional de Física da Matéria Condensada & $2009-2011-2013-2015-2017-2019$ \\
\hline $\begin{array}{l}\text { International Symposium on Lepton Photon Interac- } \\
\text { tions at High Energies }\end{array}$ & 2010 - 2012- 2014 - 2016 - 2018 - 2020 \\
\hline $\begin{array}{l}\text { International Symposium on Very High Energy Cosmic } \\
\text { Ray Interactions }\end{array}$ & 2005 - 2008 - 2011 - 2014 - 2017 \\
\hline $\begin{array}{l}\text { International Conferences on Precision Physics of } \\
\text { Simple Atomic Systems (PSAS) }\end{array}$ & $2008-2010-2012-2014-2016-2018$ \\
\hline Solvay Conference on Physics & $2008-2013-2014-2017-2019$ \\
\hline
\end{tabular}

Fonte: Dados das autoras (2021).

O primeiro evento escolhido foi o "Encontro Nacional de Física da Matéria Condensada", este evento foi escolhido por ser o evento nacional de maior reconhecimento no Brasil entre os pesquisadores da área. Este evento tem sido realizado desde 1978, sem interrupções, ao redor do Brasil.

Os dados foram recolhidos a partir dos nomes apresentados nas atas. Com estes dados foi possível criar o gráfico 1 . Neste gráfico é visível a diferença entre a quantidade de mulheres e de homens que participaram neste evento ao longo dos anos, sendo o pico de participação feminino ocorrido em 2014, contendo aproximadamente 250 mulheres, e neste mesmo ano o evento contou com um valor aproximado de 680 homens. 
Gráfico 1: Participação de mulheres x homens em Encontro Nacional de Física da Matéria Condensada

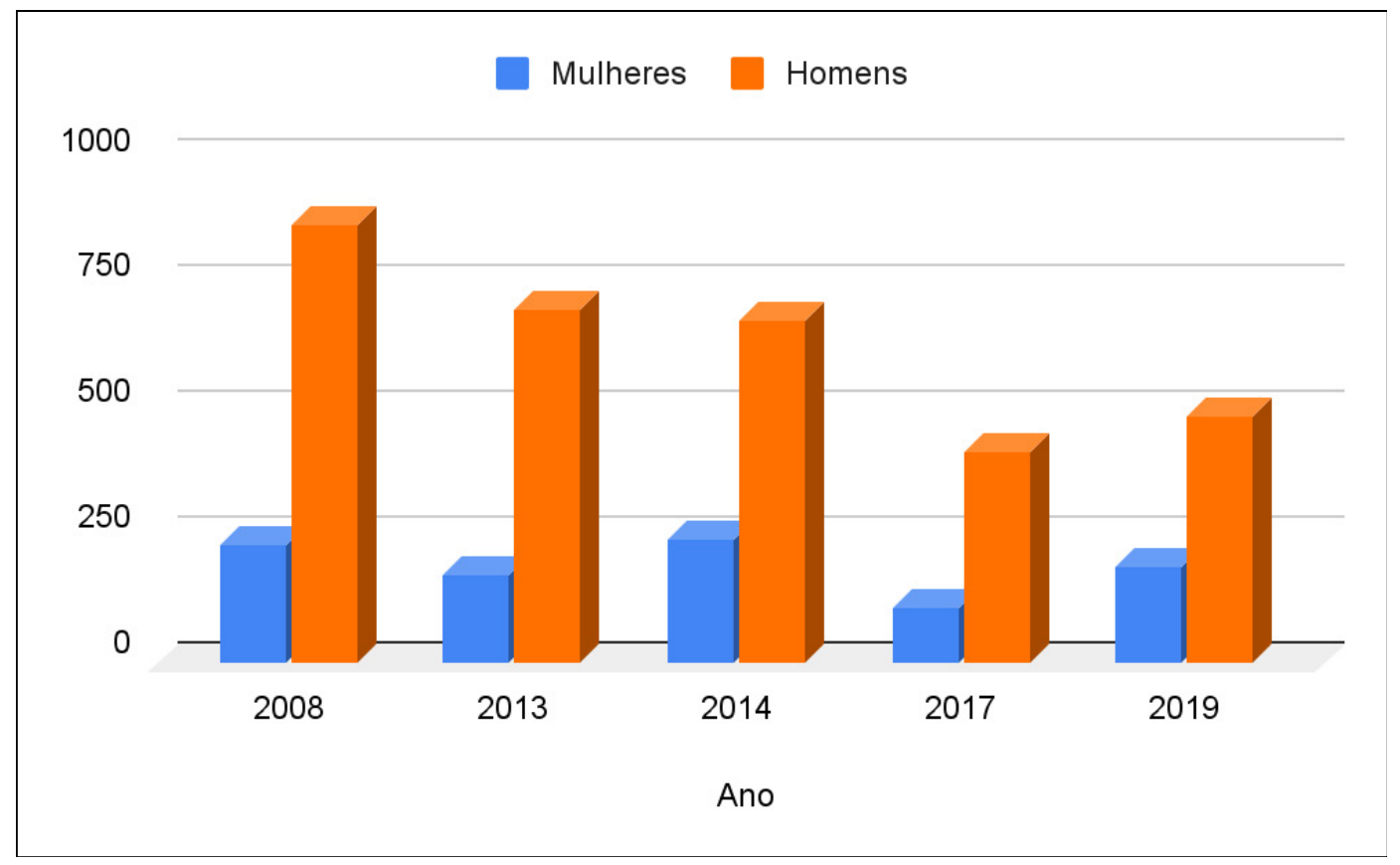

Fonte: Dados das autoras (2021).

O segundo evento escolhido, "International Symposium on Lepton Photon Interactions at High Energies", ocorre a cada 2 anos em um país diferente. Tal evento surgiu da Union of Pure and Applied Physics (IUPAP) e físicos de todo o mundo se reúnem para discutir os últimos avanços na física de partículas, Astrofísica, Cosmologia e planejar grandes instalações futuras. Os dados encontrados neste evento estão disponíveis no gráfico 2 . Ao compararmos este gráfico com 1 , percebemos que os dados não são muito diferentes, basta comparar o máximo de mulheres que participaram do evento, 67 em 2019, e o mínimo de homens já participaram do evento, 117 em 2009, ou seja o máximo de mulheres que já participaram no evento não chega perto da quantidade mínima de homens. 
Gráfico 2: Participação de mulheres $\mathrm{x}$ homens em International Symposium on Lepton Photon Interactions at High Energies

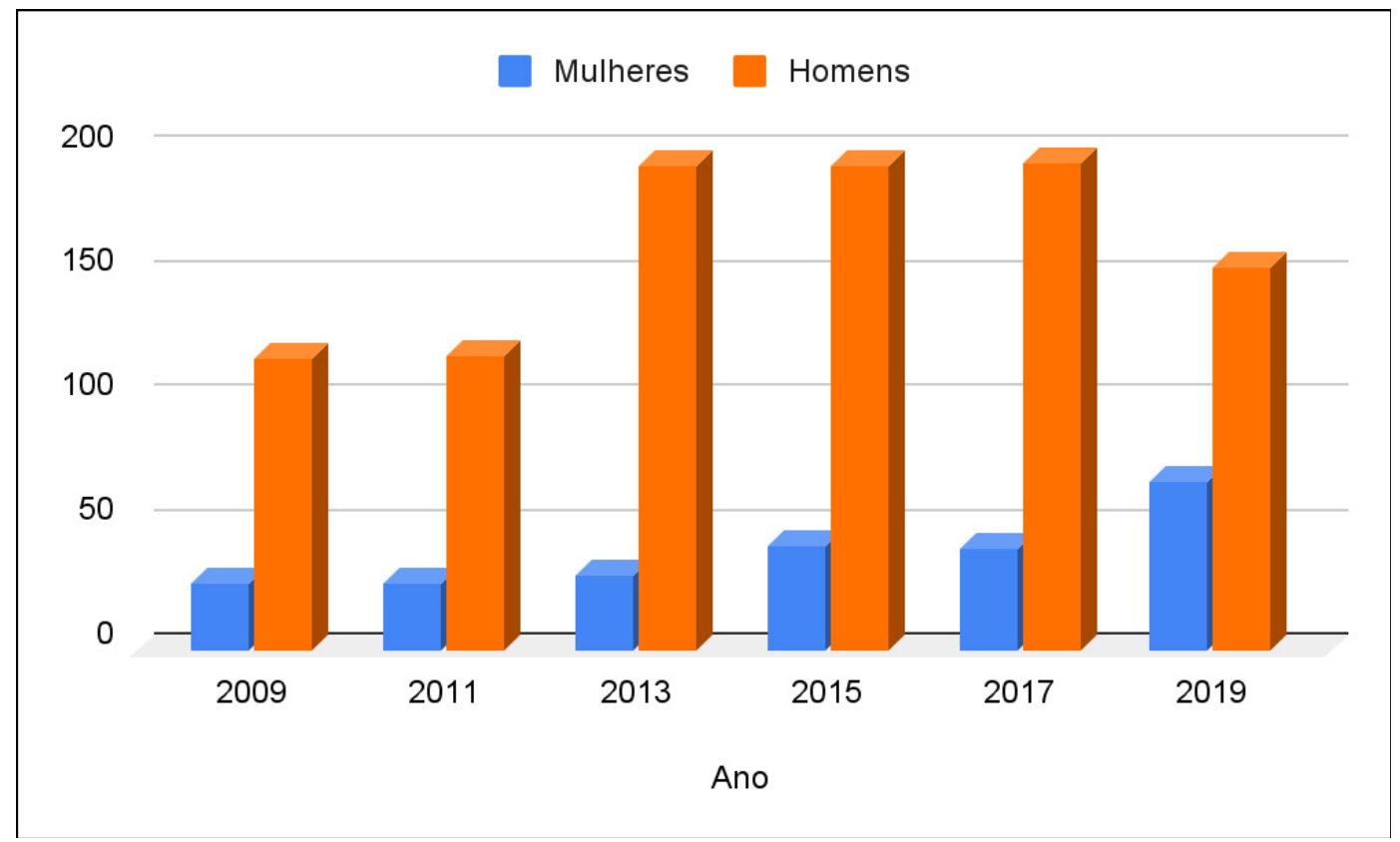

Fonte: Dados das autoras (2021).

O terceiro evento "International Symposium on Very High Energy Cosmic Ray Interactions", é um evento bienal que é apoiado pela International Union of Pure and Applied Physics (IUPAP) e a cada ano, o evento acontece em um país diferente. Este evento foi criado com o objetivo de fornecer um fórum para a discussão de interações de energia muito alta para a interpretação de dados de raios cósmicos com base nas informações disponíveis de experimentos de aceleradores e raios cósmicos. Ao analisar o gráfico 3, criado com os dados retirados deste evento, podemos perceber que está na mesma perspectiva dos gráficos anteriores, do qual 17 é a maior quantidade de mulheres que já participou desse evento, em contraste com os homens cujo mínimo foi maior que 50 . 
Gráfico 3: Participação de mulheres x homens em International Symposium on Very High Energy Cosmic Ray Interactions

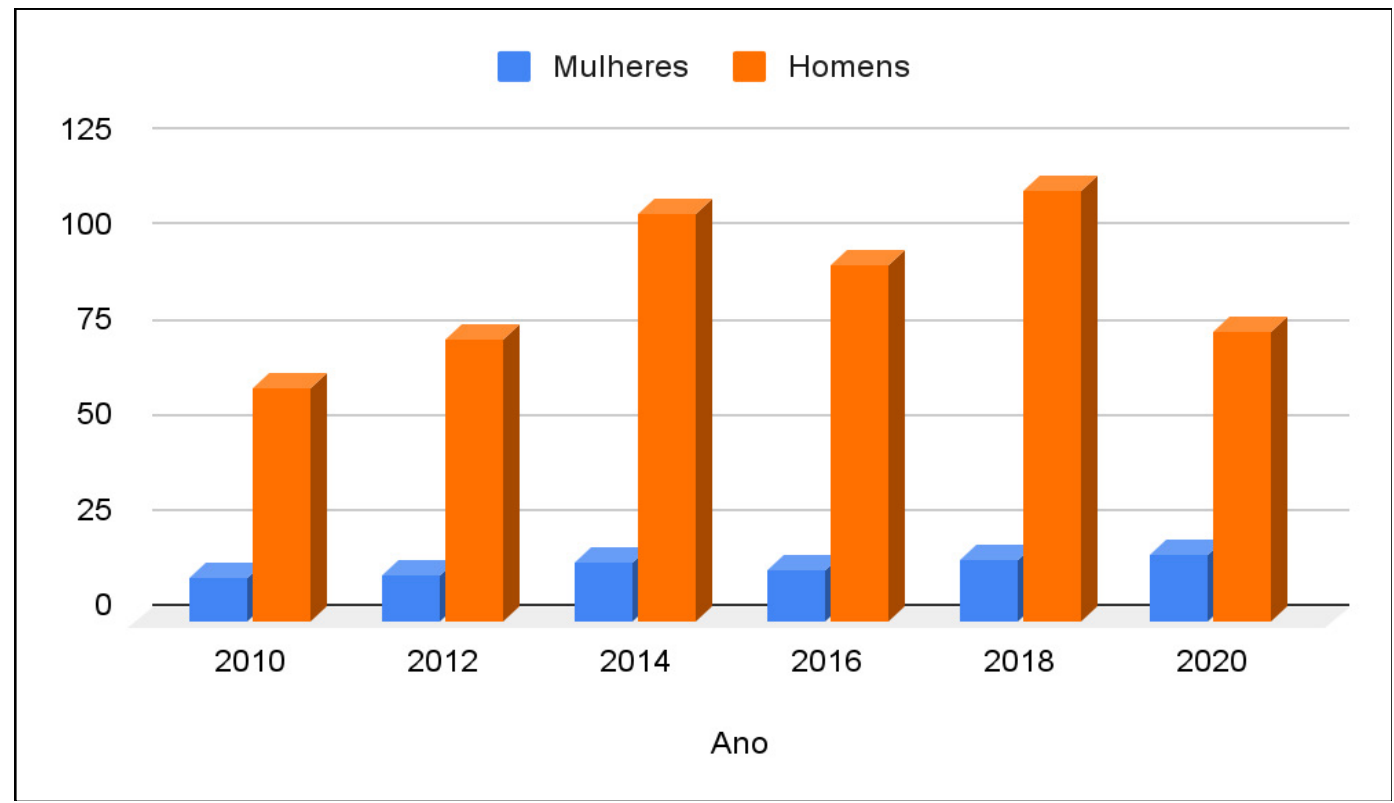

Fonte: Dados das autoras (2021).

O evento International Conferences on Precision Physics of Simple Atomic Systems, o quarto evento escolhido, é realizado a cada dois anos. O evento é dedicado a estudos de precisão de sistemas atômicos e moleculares simples, a fim de desenvolver novas abordagens e acessar várias questões fundamentais dentro e além da física atômica e possíveis novas físicas.

Ao analisar o gráfico 4, construídos dos dados retirados desse evento, o contraste entre a quantidade de mulheres e homens que participaram do evento continua evidente, em que a quantidade de mulheres não chega nem perto da quantidade de homens que participaram. Sendo o máximo de mulheres menor que 25 e o mínimo de homens perto de 50 . 
Gráfico 4: Participação de mulheres x homens em International Conferences on Precision Physics of Simple Atomic Systems

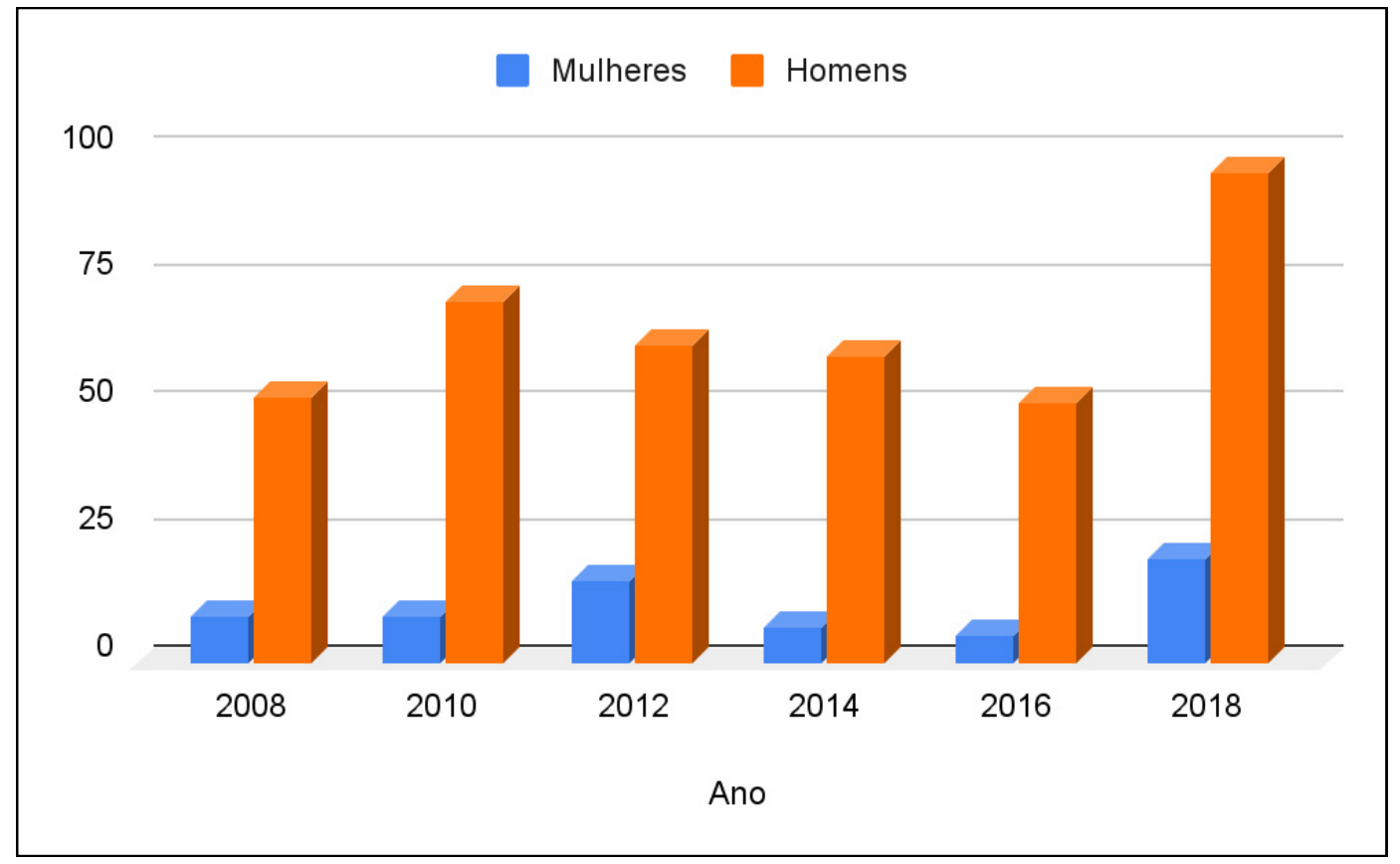

Fonte: Dados das autoras (2021).

O último evento, "Solvay Conference on Physics", é o evento de Física mais conhecido mundialmente, pois nele foi tirada a foto com os mais renomados cientistas, como Marie Curie, Albert Einstein, Max Planck, Niels Bohr, entre outros. Mesmo sendo o mais conhecido, é um evento fechado, em que os participantes são selecionados pelo presidente da Conferência. $\mathrm{O}$ máximo de mulheres que foram convidadas a participar deste evento foram 10, e o mínimo de homens 48 , como mostrado no gráfico 5 . 
Gráfico 5: Participação de mulheres x homens em Solvay Conference on Physics.

\section{Mulheres \\ Homens}

80

60

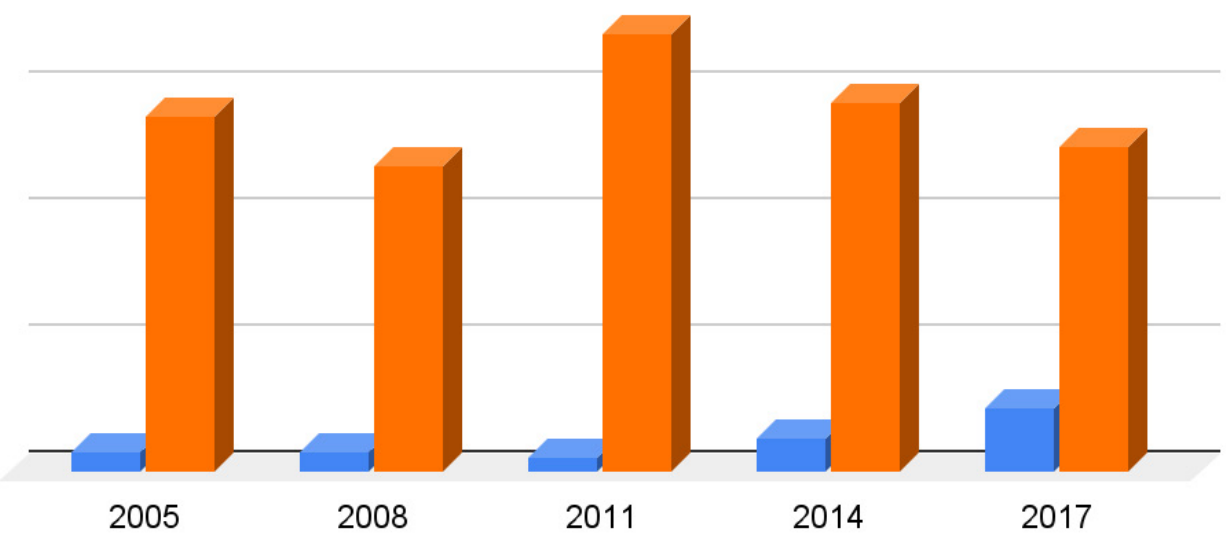

Ano

Fonte: Dados das autoras (2021).

Uma provável explicação, para o fato da baixa participação das mulheres nesse evento em específico, pode ser, devido a Conferências Solvay ser apenas para convidados, reunindo um número limitado de especialistas reconhecidos para debater um tema específico. Dessa forma, como apontou Benedito (2019) por vezes, as mulheres são responsáveis por grandes pesquisas, mas acontece o "Efeito Matilda”, assim as pesquisas realizadas por mulheres são menos reconhecidas do que as produzidas por homens. Ou ainda como a análise histórica tem nos mostrado, as mulheres são reduzidas a colaboradoras e o nome de destaque na pesquisa é masculino.

Análise dos gráficos nos revela que a participação das mulheres em eventos vem crescendo a passos lentos. Mas isso, não é uma regra, em alguns eventos como o "Encontro Nacional de Física da Matéria Condensada" e o "International Conferences on Precision Physics of Simple Atomic Systems", ocorreram quedas na participação das mulheres em determinados anos. Muitas podem ter sido as causas para a instabilidade do número de participações femininas nesses eventos, talvez uma delas pode ter sido a questão das datas de submissão dos trabalhos. 
De forma geral, todos os gráficos mostram uma alta discrepância dos dados, revelando que a quantidade de mulheres que participam dos eventos é bastante inferior aos homens, totalizando, dentro dos 12 anos analisados, 5.322 publicações masculinas em anais de evento, contra 1.349 femininas. Ou seja, os homens publicam cerca de quatro vezes mais que as mulheres.

O relatório publicado pela editora científica Elsevier, "Gender in the global research landscape" (2017) analisou o gênero das pesquisas, em conjunto com 12 países. Fazem parte das conclusões produzidas neste relatório, o fato das mulheres possuírem menor mobilidade internacional que os homens. Em consequência disso, elas podem também restringir sua rede de colaboração internacional. Dessa forma, se a colaboração internacional ocorrer com menor frequência para mulheres suas redes podem permanecer pequenas e isso pode afetar negativamente as oportunidades para progressão na carreira de pesquisadoras, e consequentemente no número de publicações de pesquisas em eventos científicos e revistas.

Mas para visualizar um panorama ainda maior da representatividade das mulheres na Física, é necessário analisar também, a participação das mulheres nas publicações em revistas de alto impacto.

\section{Participação das mulheres nas publicações em Revistas}

Na sequência deste estudo, para compreendermos se houve um crescimento na participação das mulheres nas publicações de alto impacto na área da Física, investigamos as seguintes revistas distribuídas no Quadro 3 a seguir. Investigamos as publicações dos anos de 2008 a 2020 .

Quadro 3: Revistas utilizadas na investigação.

\begin{tabular}{|l|c|}
\hline \multicolumn{1}{|c|}{ Revistas } & Qualis \\
\hline Brazilian Journal of Physic & B1 \\
\hline Reviews of modern Physic & A1 \\
\hline Physical Review $X$ & A1 \\
\hline Annalen der Physik & A2 \\
\hline Nature Physics & A1 \\
\hline
\end{tabular}

Fonte: Dados das autoras (2021). 
A primeira revista escolhida é a mais famosa revista do Brasil se tratando de Física, sendo esta Braziliam Journal of Physics. Esta revista foi fundada em 1971, e apresenta pesquisas originais e atuais em todos os aspectos, sendo experimental, teórico ou física computacional, além de ser uma revista que é reconhecida no Brasil e internacionalmente. Mesmo sendo uma revista reconhecida, o aumento na participação das mulheres está ocorrendo de forma muito lenta ao passar dos anos, aparentemente estando estagnado entre 10 á 40 o número de mulheres que contribuíram para a revista.

Gráfico 6: Participação de mulheres x homens na revista Brazilian Journal of Physics. Fonte:

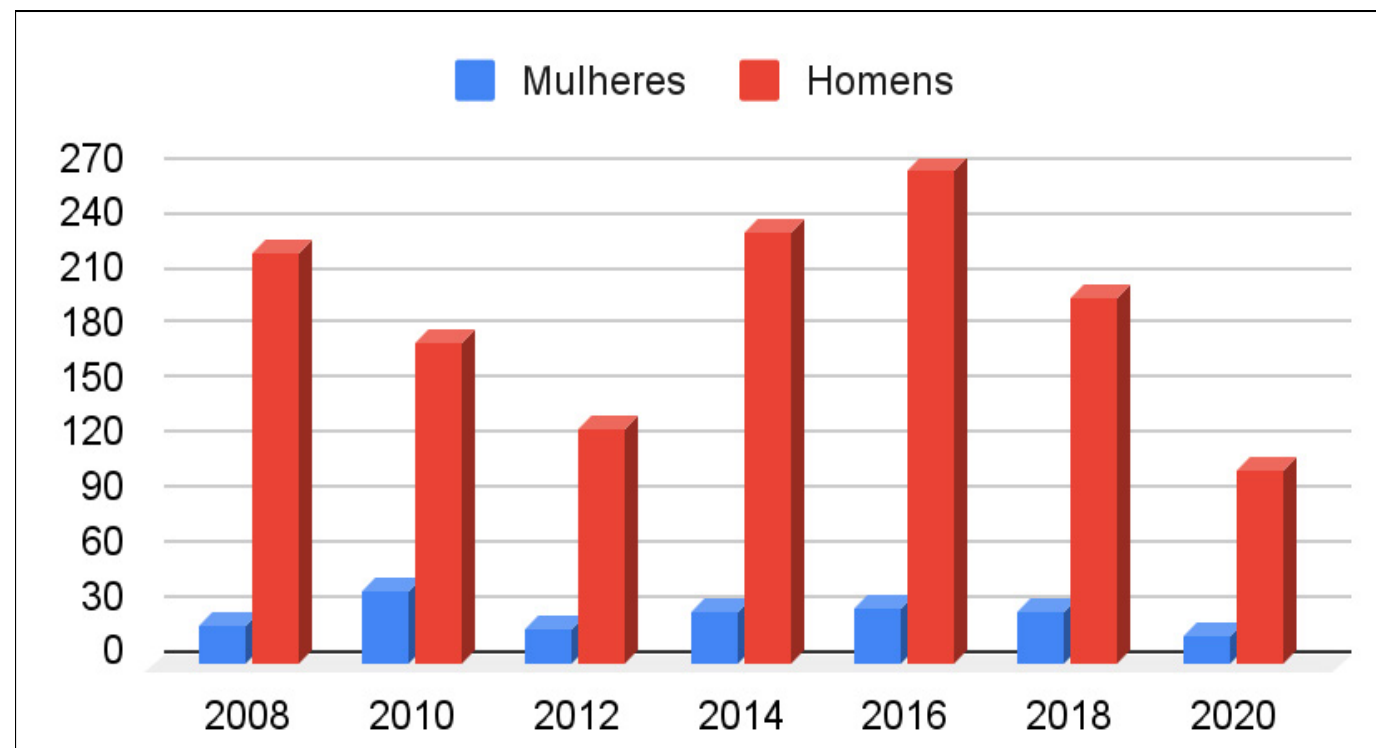

Ano

Fonte: Dados das autoras (2021).

A segunda revista escolhida iniciou suas publicações em 1929 pela American Physical Society, e é umas das revistas de física mais premiadas mundialmente e acompanhou o progresso da física durante um período de crescimento explosivo neste campo. O gráfico desta revista, gráfico 7, não é muito diferente do gráfico 6 com um aumento lento na participação das mulheres. 
Gráfico 7: Participação de mulheres x homens na revista Reviews of Modern Physics

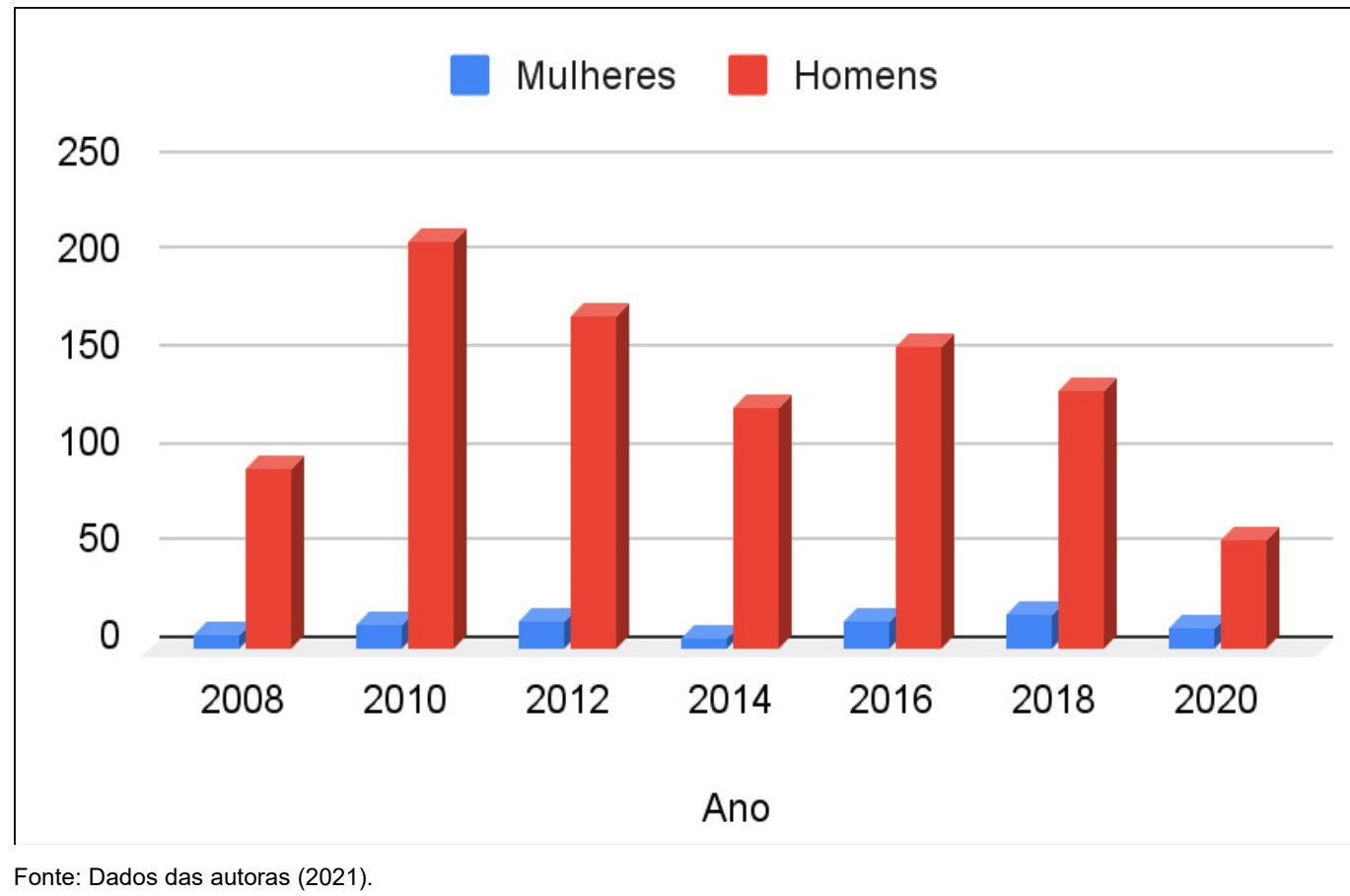

A revista Physical review X é publicada pela American Physical Society e iniciou suas publicações em 2011, sendo a revista mais recente escolhida. Diferente das outras, é um periódico somente online de acesso totalmente aberto que valoriza a inovação, a qualidade e o impacto de longo prazo na ciência que publica. Sendo uma revista de acesso mais fácil, a quantidade de mulheres que contribuíram é significativamente maior que as vistas anteriormente, sendo que 151 contribuíram com trabalhos para as publicações em 2018. Porém, ao visualizar o gráfico 8, é perceptível que os dados não fogem do padrão apresentado nas revistas anteriores, com uma quantidade de homens muito superior que a das mulheres e um lento aumento na participação das mulheres ao longo dos anos. 
Gráfico 8: Participação de mulheres x homens na revista Physical Review X.

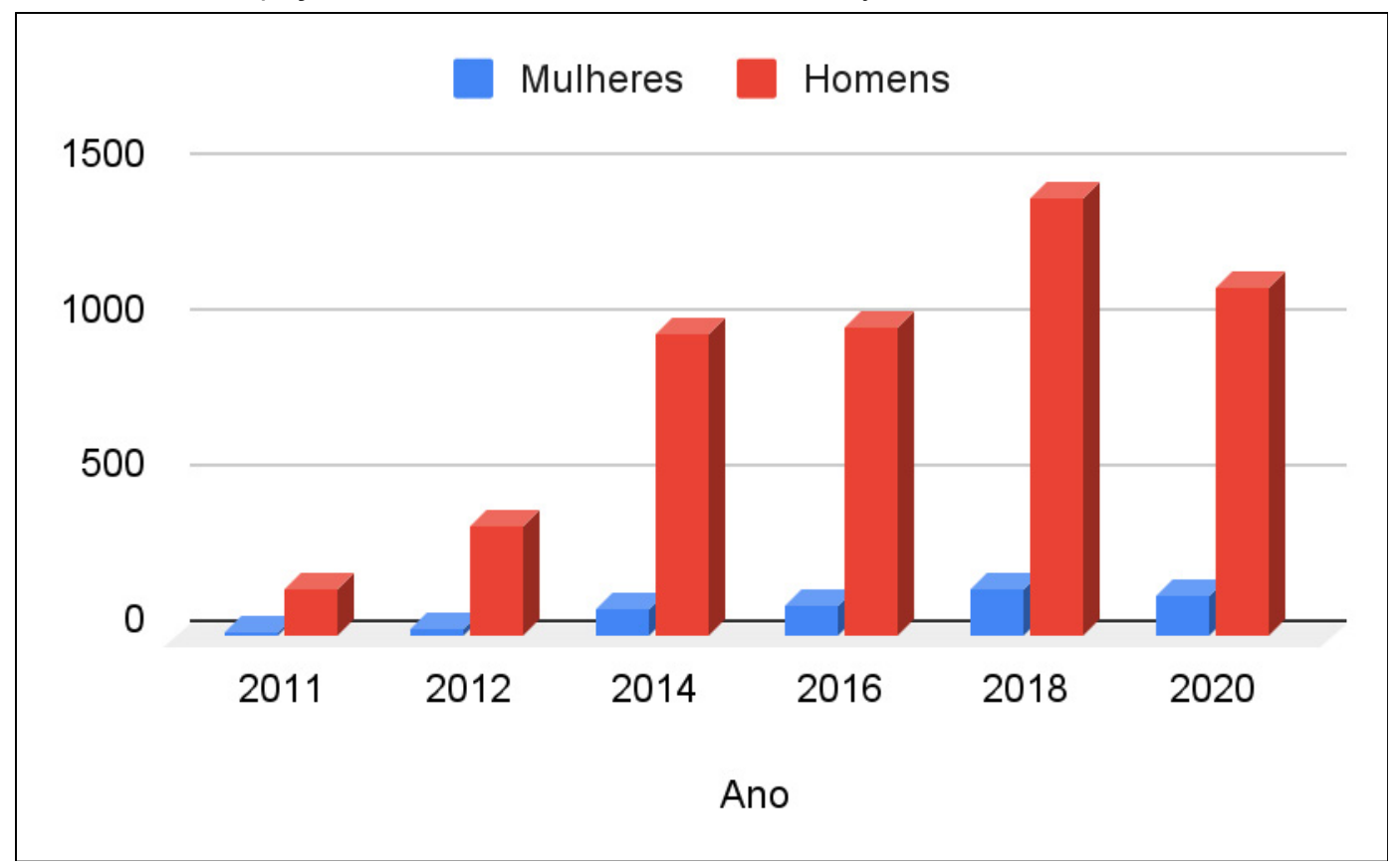

Fonte: Dados das autoras (2021).

Annalen der Physik é um dos mais conhecidos e antigos periódicos sobre física, editado em Berlim, Alemanha e começou suas publicações em 1799. A revista publica artigos originais na área da física experimental, teórica, aplicada e matemática e em áreas relacionadas. Ao analisar o gráfico 9, desta revista pode se perceber que deste 2016 houve um aumento significativo nas contribuições das mulheres, diferente dos gráficos anteriores. 
Gráfico 9: Participação de mulheres x homens na revista Annalen der Physik.

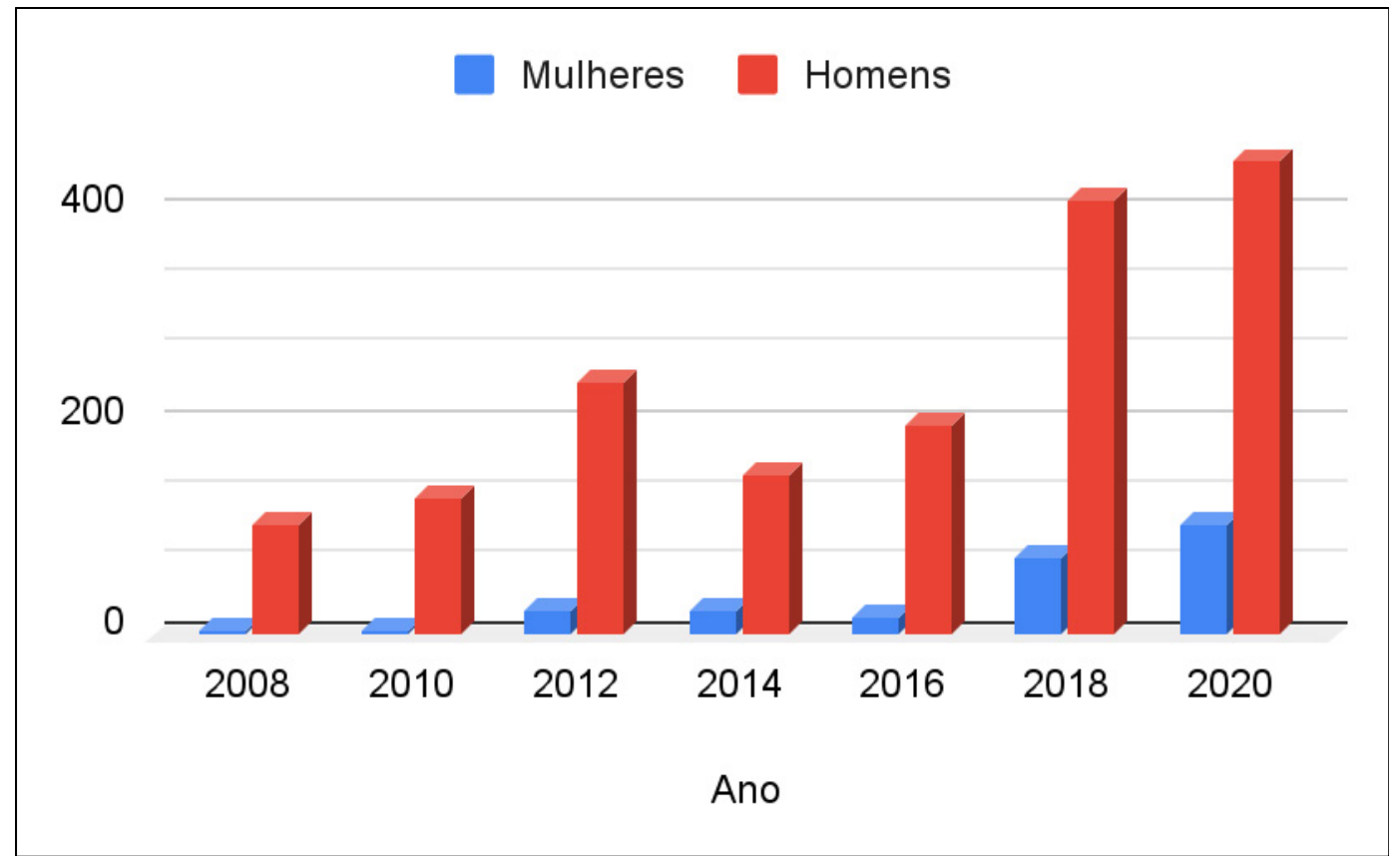

Fonte: Dados das autoras (2021).

A última revista escolhida, Nature Physics, é uma revista científica mensal publicada pela Nature Research e foi publicada pela primeira vez em 2005. Esta revista apresenta pesquisas da mais alta qualidade em todas as áreas da física, pura e aplicada. Ao observar o gráfico 10, é possível visualizar um aumento na quantidade de contribuições das mulheres nesta revista desde 2010. 
Gráfico 10: Participação de mulheres x homens na revista Nature Physics

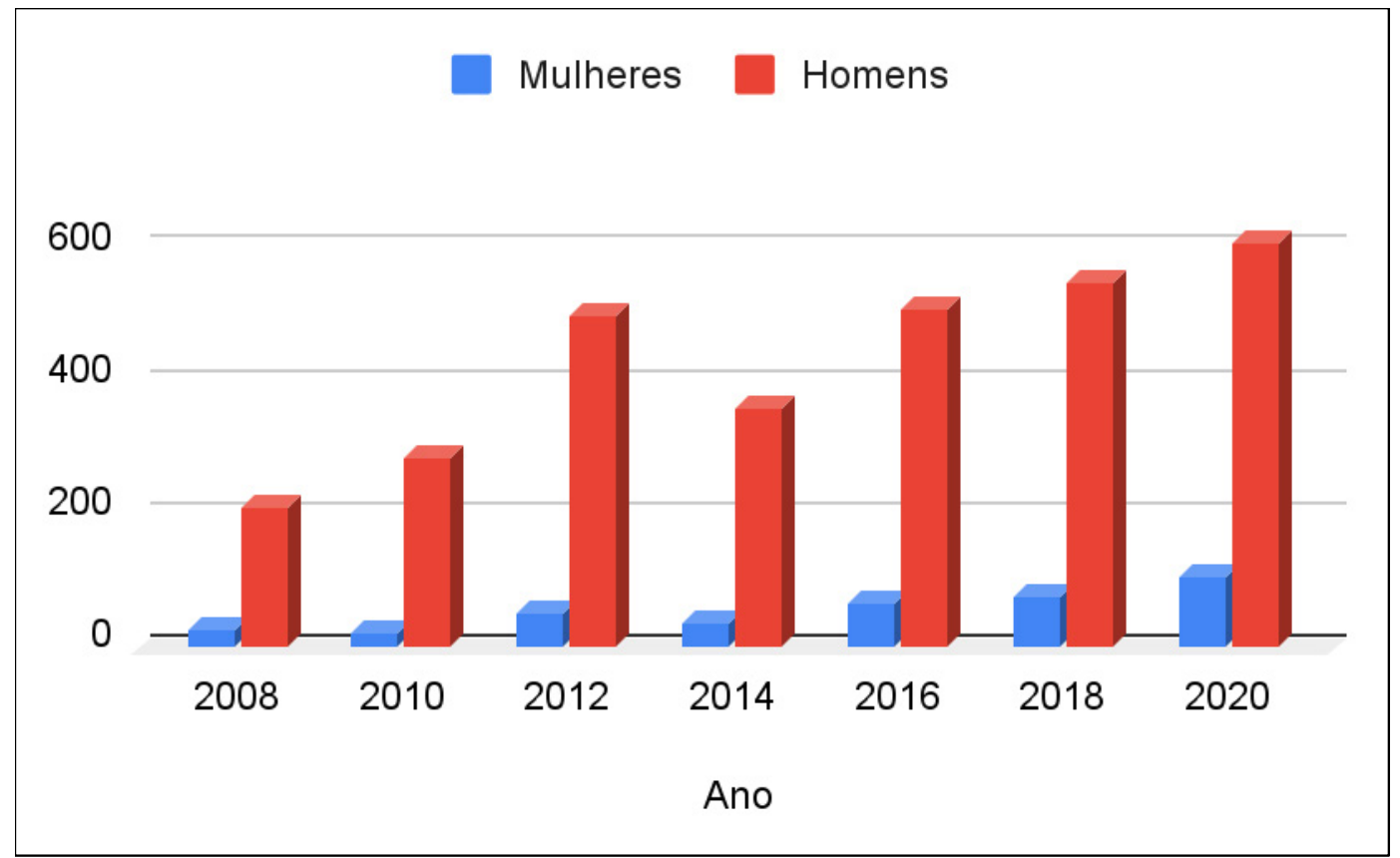

Fonte: Dados das autoras (2021).

De forma geral, podemos perceber através dos gráficos que ainda existe uma grande diferença entre a quantidade de artigos publicados em revista entre os sexos feminino e masculino, embora em algumas revista a situação, a passos lentos, venha mudando. As mulheres publicaram cerca 1.400 artigos em revistas, em contrapartida, o número de publicações masculinas se aproxima de 12.000 artigos, dentro dos 12 anos investigados nessa pesquisa. A análise dos dados confirma a chamada exclusão horizontal na área da Física, ou seja, as mulheres estão sub-representadas neste campo (LIMA, 2008).

A sub-representatividade de mulheres na área da Física não é exclusividade do Brasil, como aponta a pesquisa realizada pela International Union of Pure and Applied Physics (IUPAP) (2015). A pesquisa revela dados mundiais que mostram que o percentual de mulheres na Física diminui à medida que avançam na carreira.

Em uma pesquisa desenvolvida por Saitovitch, Lima e Barbosa (2015, p. 5) com o objetivo de analisar o percentual de mulheres bolsistas em diferentes modalidades, são apresentados resultados que chamam a atenção. As autoras verificaram 
que o número de bolsistas do sexo feminino de IC tem aumentado, mas o mesmo não acontece com as mulheres bolsistas de mestrado e doutorado "os dados mostram percentuais em torno de $21 \%$ para o mestrado e de $17 \%$ para o doutorado sem que nenhuma tendência de aumento seja detectada". A pesquisa desenvolvida por Menezes (2015) revela resultados semelhantes: apenas 11\% das mulheres são bolsistas CNPQ.

Por outro lado, Menezes et al.(2017) ao analisar os indicadores mais relevantes na distribuição de bolsas em cada nível, indica que não existe diferença considerável entre os gêneros, apesar das discrepâncias geradas por tamanhos de amostras muito distintos serem grandes.

\section{Algumas considerações acerca deste trabalho}

A participação feminina na Física é muito importante, como apontado por Barbosa (2010), a Física do século XXI necessita das melhores mentes para avançar. Dessa forma, a intenção deste trabalho é levantar dados a respeito da participação de mulheres em publicações de artigos em eventos e revistas na área da Física.

Nos eventos, em momento algum, dentro do período investigado neste estudo, o número de publicações femininas ultrapassou ou se igualou as publicações masculinas. Verificamos que mulheres vêm cada vez mais participando dos eventos, mas isso não é uma regra, nos eventos "Encontro Nacional de Física da Matéria Condensada" e o "International Conferences on Precision Physics of Simple Atomic Systems", ocorreram quedas na participação das mulheres em determinados anos. A análise geral dos dados das publicações em eventos, revelam que, dentro dos 12 anos analisados, os homens publicaram cerca de quatro vezes mais que as mulheres, são 5.322 publicações masculinas em anais de evento, contra 1.349 femininas.

Nas revistas a situação é ainda mais discrepante, nomes femininos foram encontrados em apenas 1.400 artigos, enquanto os homens publicaram cerca de 12.000 artigos. Ou seja, os homens publicaram cerca de 8,6 vezes a mais que mulheres dentro do período analisado.

Estudos têm demonstrado que a sub-representação das mulheres na área das exatas não é exclusividade do Brasil, o cenário se repete em países como Estados Unidos, França, Austrália e Canadá. Portanto ainda há muito o que fazer para alcançar a equidade de gêneros. Como apontado por Saitovitch, Lima e Barbosa (2015), 
é necessário com urgência realizar um acompanhamento do sistema para melhor identificar as barreiras que resultam na sub-representatividade das mulheres na física, e muito além de identificá-las, é preciso propor estratégias com o objetivo de superá-las, contribuindo para uma participação maior e mais qualificada das mulheres na física brasileira.

Por isso, nosso próximo passo é investigar mais a fundo essa situação, tentando descortinar o que está por trás dessa baixa participação feminina na pesquisa científica da área da Física e seu impacto na produção do conhecimento científico.

Por fim, uma pergunta que nos fizemos ao final deste trabalho foi a respeito das possíveis diferenças que podem haver entre as publicações e participações das mulheres em eventos entre a área das Ciências Exatas e das Ciências Humanas, em especial na área de Ensino.

\section{The invisibility of women in physics: a cut in the last 12 years in the production of high impact events and magazines}

\section{Abstract}

The participation of women in scientific careers has been a very recurrent theme in current studies. Research has shown that, in certain areas, female participation has increased and even surpassed men, but this does not apply to Physics. One of the challenges for the greater representation of women in the career of Physics is caused by being an area considered essentially masculine by many, which results in the demotivation of girls to follow in this field. We understand that one of the ways to change this worldview about women in physics is the need for greater representativeness in journals or scientific events in the area. This article makes a review in order to verify if there was, in the last 12 years, from 2008 to 2020, some increase in this representation. For this, five high-impact magazines and five national and international events were selected. The data collected reveal a very high discrepancy in the amount of publication between men and women, reaching cases, for example, in which men published 1,128 more journal articles than women in a single year. Therefore, this work demonstrates that there is still a lot to be done in the field of Physics so that there is equity in scientific production and female participation in publications in the área.

Keyword: Women; Physics, Publications. 


\section{Refernciais}

AGRELLO, Deise A.; GARG, Reva. Mulheres na Física: poder e preconceito nos países em desenvolvimento. Revista Brasileira de Ensino de Física, n. 31, v. 1, p. 1305-1311, abr./mai 2009.

ALMEIDA, Aline Alves. Sob o Peso do Gênero: análise da (in)visibilidade das mulheres da Física no livro didático do Ensino Médio. Patos: UEPB. Trabalho de Graduação de Curso (Graduação em Física), Centro de Ciências Exatas e Sociais Aplicadas, Universidade Estadual da Paraíba, 2017.

ANDRADE, Francisco Leal de. Determinismo Biológico e Questões de Gênero: representações e práticas de docentes do Ensino Médio. UFBA, 2011. Dissertação (Mestrado), Programa de Pós-Graduação em Ensino, Filosofia e História das Ciências, Universidade Federal da Bahia, 2011.

BARBOSA, Marcia. Equity for women in physics. Physics World, v. 16, n.7, jul. 2003.

BLAY, Eva A.; Conceição, Rosana R. da. A mulher como tema nas disciplinas da USP. Cadernos de Pesquisa, São Paulo, n. 76, p. 50-56, fev 1991.

BOCCATO, Vera Regina Casari. Metodologia da Pesquisa Bibliográfica na área Odontológica e o artigo científico como forma de comunicação. Revista de Odontologia da Universidade Cidade de São Paulo, v. 18, n. 3, p. 265-74, set-dez 2006.

BOLZANI, Vanderlan da Silva. Mulheres na ciência: por que ainda somos tão poucas?. Ciência e Cultura, São Paulo, v. 69, n. 4, p. 56-59, out./dez. 2017.

BOMBARDIERI, Marcella. Summers' remarks on women draw fire. The Boston Globe. Disponível em: <http://archive.boston.com/news/local/articles/2005/01/17/summers_remarks_on_women_draw_fire/>. Acesso em: 28 jun 2021.

CARTAXO, Sandra Maria Carlos. Gênero e Ciência: um estudo sobre as mulheres na Física. Campinas: UNICAMP, 2012. Tese (Mestrado em Política Científica e Tecnológica), Instituto de Geociências, Universidade Estadual de Campinas, 2012.

CHASSOT, Attico. A CIÊNCIA É MASCULINA? É, sim senhora!. Revista Contexto \& Educação, v. 19, n. 71-72, maio 2020.

CORDEIRO, Marinês Domingues. Mulheres na Física: um pouco de história. Caderno Brasileiro de Ensino de Física, v. 34, n. 3, p. 669-672, dez. 2017.

ELSEVIER RESEARCH INTELLINGENCE. Gender in the global research landscape: analysis of research performance through a gender lens across 20 years, 12 geographies, and 27 subject areas. Elsevier, 2017. Disponível em: <https:/www.elsevier.com/_data/assets/pdf_ file/0008/265661/ElsevierGenderReport_final_for-web.pdf>. Acesso em: 28 jun 2021.

GIBBS, Graham. Análise de dados qualitativos. São Paulo: Editora S. A., 2009.

GINITY, Eliane Goulart Mac. Imagens de mulheres nos Livros Didáticos de História. Revista do Laboratório de Ensino de História da Educação da UFRGS, v.2, n.3, p.915-932, dez. 2015.

LIMA, Betina Stefanello. Teto de vidro ou labirinto de cristal? As margens femininas da Ciência. Brasília: UnB. Trabalho de Pós-Graduação (Pós-Graduação em História), Instituto de Humanas, Universidade de Brasília, 2008.

MARTINS, Eliecília de F.; HOFFMANN, Zara. Os papéis de gênero nos livros didáticos de ciência. Revista Ensaio, v. 9, n. 1, p. 132-151, jan-jun 2007. 
MENEZES, Débora P. Editorial Mulheres na Física: a realidade em dados. Caderno Brasileiro de Ensino de Física, v. 34, n. 2, p. 341-343, ago. 2017.

MENEZES, D. P.; BRITO, C.; BUSS, K.; ANTENEODO C. Bolsistas de produtividade em pesquisa em Física e Astronomia: análise quantitativa da produtividade científica de homens e mulheres. Grupo de trabalho de Genêro da SBF, 2016. Disponível em: <http://www1.fisica.org.br/gt-genero/images/arquivos/Apresentacoes_e_Textos/dados_CNPq_2016_vf.pdf>. Acesso em: 11 Jun 2021.

NUNES, J. M.; BATISTA, R. A. da S.; PIMENTEL, W. L.; OLIVEIRA, J. F. de; COSTA, L. F. S. da; SILVA, A. dos R. "Você conhece uma cientista?": investigação temática sobre a ausência da história das mulheres na ciência no ensino básico da cidade de Castanhal - PA. Brazil Journal of Development, v. 6, n. 11, p. 86211-86221, nov. 2020.

ROSSITER, Margaret W. The Mathew Matilda Effect in Science. Social Studies of Science, v. 23, n. 2, p. 325-341, mai 1993.

SAITOVITCH, Elisa B. et al. Mulheres na Física: uma análise quantitativa. In: SAITOVITCH, Elisa B. (Org.). Mulheres na Física: Casos históricos, panorama e perspectivas. São Paulo: Editora Livraria da Física, 2015. p. 245 - 256.

SCHIEBINGER, Londa. Mais mulheres na ciência: questões de conhecimento. História, ciência, saúde-Manguinhos. v.15, suplemento, p.269-281, jun. 2008. Disponível em: <https://www.scielo. br/j/hcsm/a/LZcRqYbsQR4cxYkgfCGyjyr/?lang=pt>. Acesso em 28 de junho de 2021.

SILVA, A. F. da; SANTOS, A. P. O. dos; HEERDT, B. Questões de gênero na educação científica: tendências nas pesquisas nacionais e internacionais. In: ANAIS DO XI ENCONTRO NACIONAL DE PESQUISA EM EDUCAÇÃO EM CIÊNCIAS, Fortaleza, 2017. XI ENPEC. Disponível em: <http://www.abrapecnet.org.br/enpec/xi-enpec/anais/resumos/R2223-1.pdf> Acesso em: 07 jul 2021.

SILVA, Fabiane F. da; RIBEIRO, Paula R. C.. Trajetórias de mulheres na ciência: "ser cientista" e "ser mulher". Ciência \& Educação, v. 20, n. 2, p. 449-466, 2014.

UFRGS TV. Palestrante: Márcia Barbosa. Física na Cultura - Mulheres na Física [3/3]. Abr. 2010. Disponível em : < https://www.youtube.com/watch?v=halxFjx3hEE> Acesso em: 28 de Jun 2021.

VASCONCELLOS, Elza C. C.; BRISOLLA, Sandra N. Presença Feminina no Estudo e no Trabalho da Ciência na Unicamp. Cadernos Pagu, n. 32., p. 215-265, 209, Jun 2009. 